\title{
Pirfenidone in Unclassifiable Interstitial Lung Disease: A Subgroup Analysis by Concomitant Mycophenolate Mofetil and/or Previous Corticosteroid Use
}

\author{
Michael Kreuter (D) - Toby M. Maher · Tamera J. Corte • \\ Maria Molina-Molina · Judit Axmann · Frank Gilberg • \\ Klaus-Uwe Kirchgaessler • Vincent Cottin
}

Received: October 1, 2021 / Accepted: November 29, 2021 / Published online: December 22, 2021

(C) The Author(s) 2021

\section{ABSTRACT}

Introduction: There are currently no approved treatments solely for unclassifiable interstitial lung disease (uILD); however, a recent trial showed this population can benefit from pirfenidone. We report a subgroup analysis of this

M. Kreuter $(\bowtie)$

Department of Pulmonology and Critical Care Medicine, Center for Interstitial and Rare Lung Diseases, Thoraxklinik, University of Heidelberg, Roentgen Street 1, 69126 Heidelberg, Germany e-mail: kreuter@uni-heidelberg.de

M. Kreuter

German Center for Lung Research, Heidelberg, Germany

\section{T. M. Maher}

Interstitial Lung Disease Unit, Royal Brompton Hospital, London, UK

\section{T. M. Maher}

Fibrosis Research Group, National Heart and Lung Institute, Imperial College London, London, UK

\section{T. M. Maher}

Hastings Center for Pulmonary Research and Division of Pulmonary, Critical Care and Sleep Medicine, Keck School of Medicine, University of Southern California, Los Angeles, CA, USA

T. J. Corte

Department of Respiratory Medicine, Royal Prince Alfred Hospital, Camperdown, NSW, Australia trial to assess the effects of immunomodulators (concomitant mycophenolate mofetil [MMF] and/or previous corticosteroids) with pirfenidone in patients with uILD.

Methods: This was a multicenter, international, double-blind, randomized, placebo-controlled phase II trial of patients with progressive fibrosing uILD (NCT03099187). Patients were randomized (1:1) to receive pirfenidone

\section{T. J. Corte}

Medical School, University of Sydney,

Camperdown, NSW, Australia

M. Molina-Molina

ILD Unit, Respiratory Department, Institut

d'Investigació Biomèdica de Bellvitge (IDIBELL),

University Hospital of Bellvitge, L'Hospitalet de

Llobregat, Barcelona, Spain

\section{Molina-Molina}

Centro de Investigación Biomédica en Red Enfermedades Respiratorias (CIBERES), Madrid, Spain

J. Axmann · F. Gilberg · K.-U. Kirchgaessler

F. Hoffmann-La Roche, Ltd., Basel, Switzerland

V. Cottin

National Reference Coordinating Center for Rare Pulmonary Diseases (OrphaLung), Louis Pradel Hospital, Hospices Civils de Lyon, Lyon, France

V. Cottin

IVPC, INRAE, Member of ERN-LUNG, University of Lyon, Lyon, France 
$2403 \mathrm{mg} /$ day or placebo. This analysis assessed forced vital capacity (FVC) change from baseline measured using site spirometry (key secondary endpoint) and safety over 24 weeks by concomitant MMF use at randomization (prespecified analysis) and/or previous corticosteroid use (post hoc analysis).

Results: Overall, 253 patients were randomized, including $45(17.8 \%)$ patients (pirfenidone, $n=23$; placebo, $n=22$ ) receiving concomitant MMF with/without previous corticosteroids (MMF subgroup); 79 (31.2\%) patients (pirfenidone, $n=44$; placebo, $n=35$ ) receiving previous corticosteroids without MMF (corticosteroids/no-MMF subgroup); and 129 (51.0\%) patients (pirfenidone, $n=60$; placebo, $n=69$ ) not receiving concomitant MMF or previous corticosteroids (no-corticosteroids/noMMF subgroup). At 24 weeks, difference in mean $(95 \%$ confidence interval) FVC change from baseline between pirfenidone and placebo was $-55.4 \mathrm{~mL}(-206.7,96.0 ; P=0.4645)$ in the MMF subgroup; $128.4 \mathrm{~mL}(-6.4,263.3$; $P=0.0617)$ in the corticosteroids/no-MMF subgroup; and $115.5 \mathrm{~mL} \quad(35.1, \quad 195.9$; $P=0.0052)$ in the no-corticosteroids/no-MMF subgroup. All subgroups generally exhibited a similar pattern of treatment-emergent adverse events.

Conclusion: Although limited by design and small sample sizes, this analysis suggests pirfenidone may be less effective in patients with UILD receiving concomitant MMF, whereas a beneficial treatment effect was observed in patients not receiving concomitant MMF regardless of previous corticosteroid use. Pirfenidone was well tolerated regardless of MMF and/or corticosteroid use.

Trial Registration Number: ClinicalTrials.gov: NCT03099187.

Keywords: Corticosteroid; Immunomodulator; Mycophenolate mofetil; Pirfenidone; Unclassifiable interstitial lung disease

\section{Key Summary Points}

Why carry out this study?

Diagnosis of a specific interstitial lung disease (ILD) is important for identifying the most appropriate management strategy and informing disease prognosis; however, despite thorough investigation by a multidisciplinary team, some patients are diagnosed with unclassifiable ILD (uILD).

There are currently no approved treatments solely indicated for uILD; however, a recent 24 -week phase II clinical trial that evaluated the efficacy and safety of pirfenidone versus placebo in patients with progressive fibrotic uILD suggested that this population of patients can benefit from pirfenidone treatment.

The subgroup analysis of this phase II clinical trial aimed to assess the effects of immunomodulators (concomitant mycophenolate mofetil [MMF] and/or previous corticosteroids) with pirfenidone in patients with uILD.

What was learned from the study?

Despite the limitation of a small sample size, this subgroup analysis suggests that pirfenidone may be less effective in patients with uILD receiving MMF at randomization, whereas a beneficial treatment effect for pirfenidone on forced vital capacity change was observed in patients not receiving MMF at randomization regardless of previous corticosteroid use; pirfenidone was well tolerated regardless of MMF and/or corticosteroid use.

Further research is needed to explore these findings in a larger group of patients in a study appropriately designed to determine the effects of concomitant immunomodulators with pirfenidone in patients with progressive uILD. 


\section{INTRODUCTION}

Interstitial lung diseases (ILDs) are a large, heterogeneous group of diseases characterized by abnormalities of pulmonary interstitium or alveoli, including fibrosis [1]. Although some ILDs have a progressive fibrosing phenotype similar to idiopathic pulmonary fibrosis (IPF), which is the most common form of idiopathic interstitial pneumonia [2], the clinical course of other ILDs is variable $[1,3,4]$.

Diagnosis of a specific ILD is important for identifying the most appropriate management strategy and informing disease prognosis $[1,3,4]$. However, despite thorough investigation by a multidisciplinary team, some patients are diagnosed with unclassifiable ILD (uILD) due to nonspecific or conflicting clinical, radiological, or histopathological findings or where invasive diagnostic procedures are inappropriate or not possible $[1,3,4]$. Adding to the heterogeneity of the UILD population is a subgroup of patients that meet criteria for the research classification scheme of interstitial pneumonia with autoimmune features (IPAF), who often fall within the category of UILD $[3,5]$.

Small retrospective cohort studies and a case study have shown potential benefits of immunomodulators including mycophenolate mofetil (MMF) and corticosteroids in some patients with IPAF [6-9] or other uILDs [10]. Moreover, immunomodulators are frequently used as concomitant medications in patients with fibrosing ILDs in clinical studies or in clinical practice [11-13].

There are currently no approved treatments solely indicated for uILD, but nintedanib is now approved for progressive fibrosing ILDs, including uILD, on the basis of its efficacy and safety demonstrated in the INBUILD study $[12,14]$. Moreover, a recent 24-week phase II clinical trial that evaluated the efficacy and safety of pirfenidone versus placebo in patients with progressive fibrotic uILD suggested that this population of patients can benefit from antifibrotic treatment with pirfenidone [15]. It should be noted that the primary endpoint (forced vital capacity [FVC] measured by home spirometry) could not be analyzed in this trial because of technical and analytical issues with home spirometry. Therefore, the beneficial effect of pirfenidone in patients with UILD was based on the analysis of the key secondary endpoints, including change in FVC measured by site spirometry, an outcome which has been utilized as a primary endpoint in clinical trials for IPF $[16,17]$ and other progressive fibrosing ILDs $[14,18]$. Moreover, pirfenidone treatment was efficacious and well tolerated in patients with other non-IPF progressive fibrosing ILDs, including connective tissue disease-associated ILD, fibrotic nonspecific interstitial pneumonia, chronic hypersensitivity pneumonitis, and asbestos-induced lung fibrosis, in the RELIEF trial [18].

Here, we report a subgroup analysis of the phase II trial of pirfenidone in patients with uILD to assess the effects of immunomodulators (concomitant MMF and/or previous corticosteroids) with pirfenidone in these patients.

\section{METHODS}

This was a multicenter, international, doubleblind, randomized, placebo-controlled phase II trial of patients with progressive fibrosing uILD (ClinicalTrials.gov: NCT03099187), the methods of which have been previously described $[15,19]$. The trial was done in accordance with the ethical principles of the Good Clinical Practice guidelines and the Declaration of Helsinki, and local laws for countries in which the research was done. Informed consent was obtained from each participant by the study investigator before any study-specific screening procedures were done.

Patients were randomized in a $1: 1$ ratio to receive pirfenidone $2403 \mathrm{mg}$ /day or placebo. Randomization was stratified by concomitant MMF use. Patients were permitted to receive a maximum MMF dose of $1.5 \mathrm{~g}$ twice daily; otherwise, the dosage was at the discretion of the investigator. Patients who were not taking MMF at the time of enrollment were not permitted to start MMF during the trial. Patients were not permitted to receive any other immunomodulators during the trial or to 
receive treatment with high-dose systemic corticosteroids (i.e., more than $15 \mathrm{mg}$ /day of prednisolone or equivalent) for more than 4 weeks during the trial.

The primary endpoint was predicted mean change in FVC (in milliliters) measured using daily home spirometry over 24 weeks. As previously reported, the primary endpoint could not be analyzed because of technical issues with the home spirometers and the inclusion of patients with a small number of readings collected within a short time window [15]. Examples of FVC readings recorded at home included daily values of less than $0.5 \mathrm{~L}$ or greater than $6 \mathrm{~L}$, and predicted increases of $33 \mathrm{~L}$ at 24 weeks, which are clearly impossible. As the statistical assumptions for applying a Student's $t$ test could not be fulfilled, the pre-specified model could not be applied to the home spirometry data [15]. Secondary endpoints included change in FVC from baseline measured by spirometry during clinic visits, change in percentage predicted carbon monoxide diffusing capacity (DLco) from baseline, and change in 6-min walk distance (6MWD) from baseline.

Primary and secondary efficacy endpoints were assessed in the intention-to-treat (ITT) population, which included all randomly assigned patients. Safety was assessed in the safety analysis set (SAS), which included all randomly assigned patients who received at least one dose of study drug. The incidence and severity of treatment-emergent adverse events (TEAEs) and withdrawals from study treatment or study discontinuations were recorded.

In this subgroup analysis, we investigated baseline characteristics, changes in FVC (in milliliters and percentage predicted) from baseline measured using site spirometry, change in percentage predicted DLco from baseline, change in 6MWD from baseline, and safety by concomitant MMF use at randomization (prespecified analysis), and/or previous corticosteroid use (all patients who started corticosteroids prior to or at baseline; post hoc analysis), all over 24 weeks. It should be noted that patients who received corticosteroids after baseline were not included in the analyses because those patients started treatment at different times and for different durations, which may have confounded the results of the analyses.

The change in FVC (percentage predicted and in milliliters) between baseline and week 24 measured by site spirometry was estimated from a linear regression model. The mean change in FVC was compared between treatment groups using a Student's $t$ test with a two-sided significance level of $5 \%$. Changes in percentage predicted FVC, percentage predicted DLco, and 6MWD were compared between treatment groups using a rank analysis of variance model, with change from baseline used as an outcome variable and standardized rank baseline value used as a covariate. Categorical changes in percentage predicted FVC (greater than 5\% and greater than 10\%) were compared between treatment groups using a Cochran-Mantel-Haenszel test. Categorical changes in percentage predicted DLco (greater than 15\%) were compared between treatment groups using logistic regression or Fisher's exact test, where appropriate. Categorical changes in 6MWD (greater than $50 \mathrm{~m}$ ) were compared between treatment groups using logistic regression.

\section{RESULTS}

Overall, 253 patients were randomized and included in the ITT analysis set (pirfenidone, $n=127$; placebo, $n=126$ ). The SAS included 251 patients (pirfenidone, $n=127$; placebo, $n=124)$. The present analysis included 45 $(17.8 \%)$ patients (pirfenidone, $n=23$; placebo, $n=22$ ) who received concomitant MMF treatment with or without previous corticosteroid treatment (MMF subgroup), 79 (31.2\%) patients (pirfenidone, $n=44$; placebo, $n=35$ ) who received previous corticosteroid treatment without MMF (corticosteroids/no-MMF subgroup), and 129 (51.0\%) patients (pirfenidone, $n=60$; placebo, $n=69$ ) who received no concomitant MMF treatment or previous corticosteroid treatment (no-corticosteroids/no-MMF subgroup). In total, 21 (91.3\%) and 17 (77.3\%) patients receiving concomitant MMF treatment also received concomitant corticosteroid treatment during the double-blind period in the pirfenidone and placebo groups, respectively. 


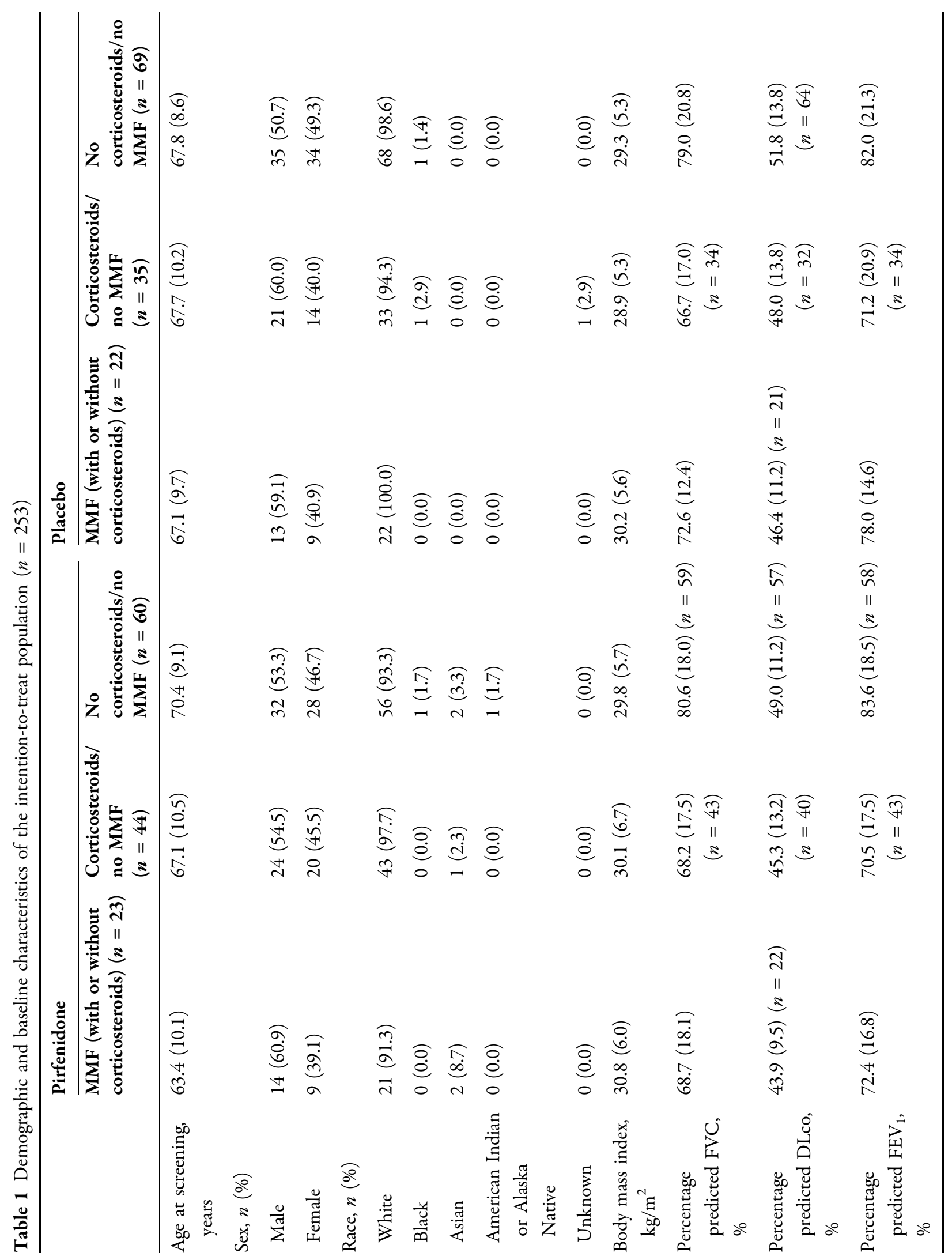




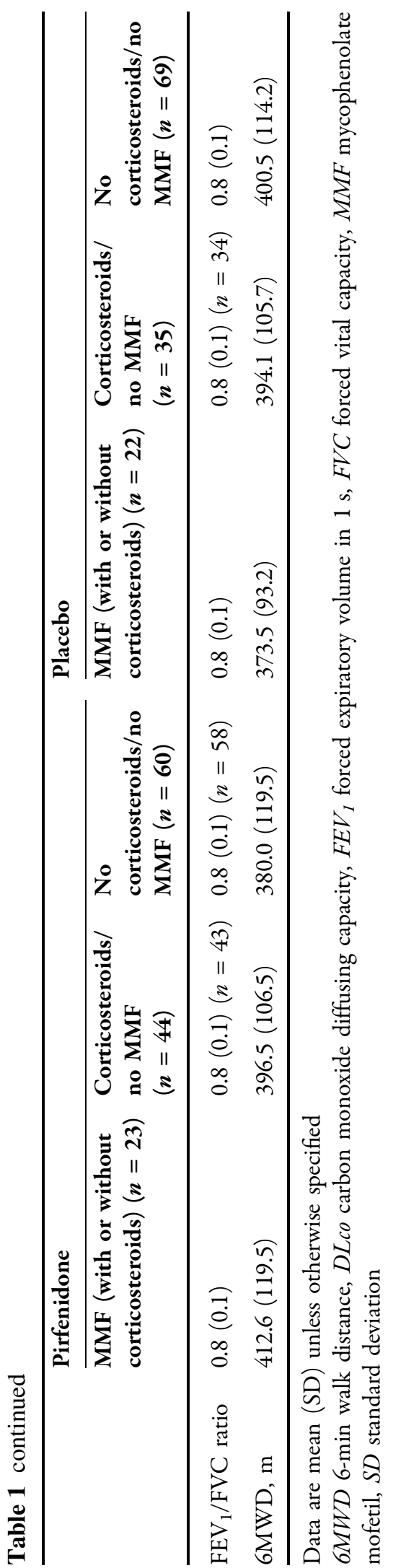

Overall, 33 patients in the study had an IPAF diagnosis (pirfenidone, $n=15$ and placebo, $n=18$ ); 12 of these patients were in the MMF subgroup (pirfenidone, $n=6$; placebo, $n=6$ ).

Demographics and baseline characteristics of the ITT population are shown in Table 1. Demographics were similar between all concomitant MMF and previous corticosteroid subgroups (mean age 63.4-70.4 years; male 50.7-60.9\%; white race 91.3-100.0\%). Mean percentage predicted FVC at baseline measured by site spirometry was similar between the MMF subgroup (68.7-72.6\%) and corticosteroids/noMMF subgroup (66.7-68.2\%) but was slightly higher in the no-corticosteroids/no-MMF subgroup (79.0-80.6\%). Mean percentage predicted DLco (43.9-51.8\%) and mean 6MWD $(373.5-412.6 \mathrm{~m})$ at baseline were similar between all concomitant MMF and previous corticosteroid subgroups.

At 24 weeks, the mean ( $95 \%$ confidence interval [CI]) predicted FVC change from baseline was $-132.8 \mathrm{~mL}(-253.2,-12.4)$ in the pirfenidone group and $-77.5 \mathrm{~mL}(-176.6$, 21.6) in the placebo group for the MMF subgroup (difference $-55.4 \mathrm{~mL}[-206.7,96.0]$; $P=0.4645) ;-16.5 \mathrm{~mL}(-100.7,67.7)$ in the pirfenidone group and $-144.9 \mathrm{~mL}(-256.7$, - 33.2) in the placebo group for the corticosteroids/no-MMF subgroup (difference $128.4 \mathrm{~mL}$ $[-6.4, \quad 263.3] ; \quad P=0.0617) ; \quad$ and $12.8 \mathrm{~mL}$ $(-54.1,79.7)$ in the pirfenidone group and $-102.7 \mathrm{~mL}(-151.5,-53.8)$ in the placebo group for the no-corticosteroids/no-MMF subgroup (difference $115.5 \mathrm{~mL}$ [35.1, 195.9]; $P=0.0052$; Fig. 1). Categorical change in FVC of greater than $5 \%$ or greater than $10 \%$, change in percentage predicted DLco, and change in 6MWD by concomitant MMF and previous corticosteroid use are shown in Table 2.

Generally, a similar pattern of TEAEs was observed across all subgroups (Table 3). The incidence of treatment-related TEAEs was slightly higher in the MMF subgroup (82.6\%) than the other subgroups (68.2-68.3\%) for pirfenidone-treated patients. The incidence of treatment-related TEAEs was lower in the corticosteroids/no-MMF subgroup (37.1\%) than in the other subgroups (49.3-50.0\%) for placebotreated patients. Additionally, higher 


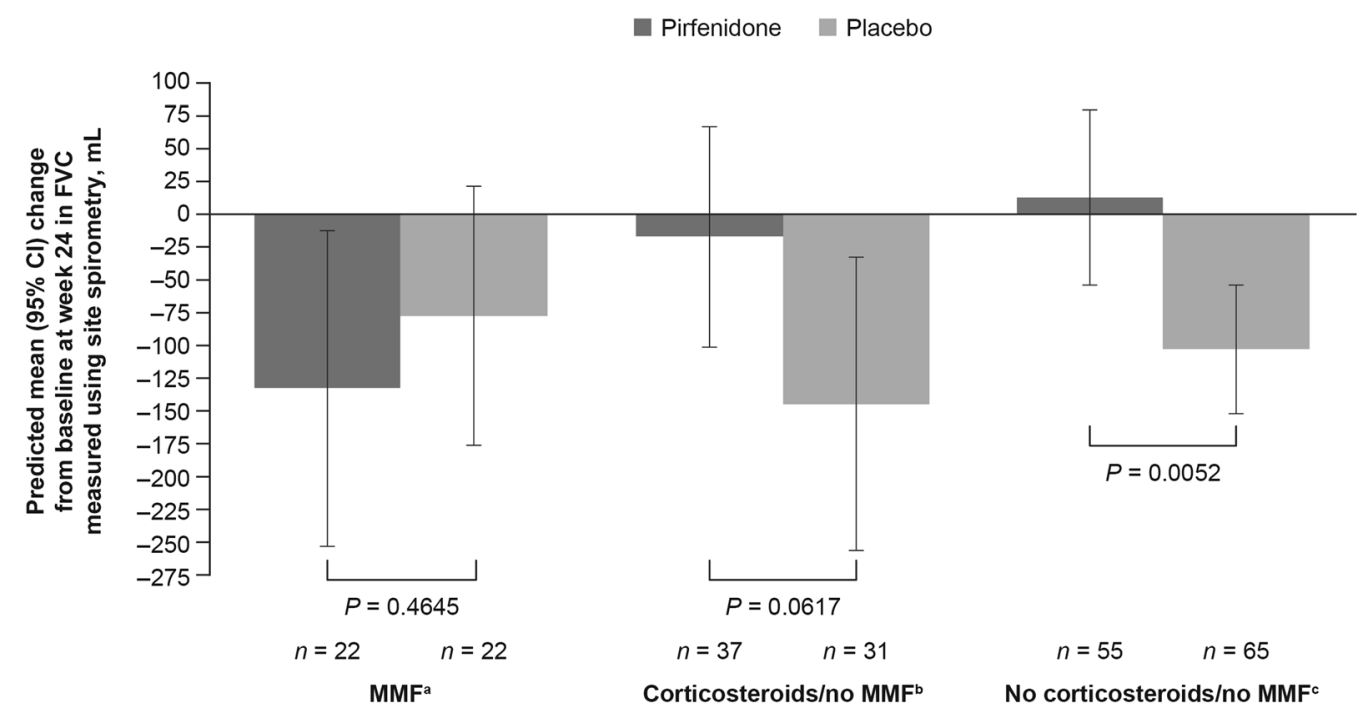

Fig. 1 Mean (95\% CI) change from baseline to week 24 in FVC (measured by site spirometry) for pirfenidone versus placebo by concomitant MMF use at randomization and/or previous corticosteroid use. FVC $(\mathrm{mL})$ decline at week 24 measured by site spirometry and estimated from linear regression model. Two-sided 95\% CI for mean value is based on percentiles of the $t$ distribution. $C I$ confidence

proportions of serious TEAEs (25.7\%) and severe TEAEs $(31.4 \%)$ were observed in the placebo group for the corticosteroids/no-MMF subgroup than in the other subgroups (serious TEAEs, 9.1-11.9\%; severe TEAEs, 9.1-19.4\%). Gastrointestinal (GI) disorders were more frequent in the MMF subgroup than in the other subgroups in both the pirfenidone $(69.6 \%$ vs $47.7-58.3 \%)$ and placebo $(54.5 \%$ vs $31.4-40.3 \%)$ groups.

\section{DISCUSSION}

Although the overall results of the uILD study suggested that patients with progressive fibrosing uILD can benefit from pirfenidone over 24 weeks, this subgroup analysis suggests that pirfenidone may be less effective in patients receiving concomitant MMF at randomization versus those not receiving MMF. A numerically beneficial treatment effect for pirfenidone versus placebo on FVC change was observed regardless of previous corticosteroid use in patients not receiving $\mathrm{MMF}$, although the interval, $F V C$ forced vital capacity, $M M F$ mycophenolate mofetil. ${ }^{2}$ Patients who received concomitant MMF at randomization with/without corticosteroids prior to or at baseline. ${ }^{b}$ Patients who received corticosteroids prior to or at baseline without concomitant MMF at randomization. ${ }^{\mathrm{c}}$ Patients who did not receive corticosteroids prior to or at baseline or concomitant MMF at randomization

beneficial effect in the corticosteroids/no-MMF subgroup was not statistically significant. This was most likely as a result of small samples sizes and because the study was not designed nor powered to show differences between corticosteroid subgroups. It is important to note that although the patient demographics and clinical characteristics were generally similar irrespective of concomitant MMF and/or previous corticosteroid use, mean percentage predicted FVC at baseline was higher in the no-corticosteroids/ no-MMF subgroup compared with the MMF and corticosteroids/no-MMF subgroups. This difference in FVC at baseline may have contributed to the lack of statistically significant effect of pirfenidone on FVC decline in the MMF and corticosteroids/no-MMF subgroups versus the no-corticosteroids/no-MMF subgroup. In general, FVC measurements can be highly variable between patients, and small sample sizes can lead to large CIs, which were also observed in these analyses. Variable FVC measurements suggest that efficacy may differ depending on patients' genotype and/or phenotype, and as 


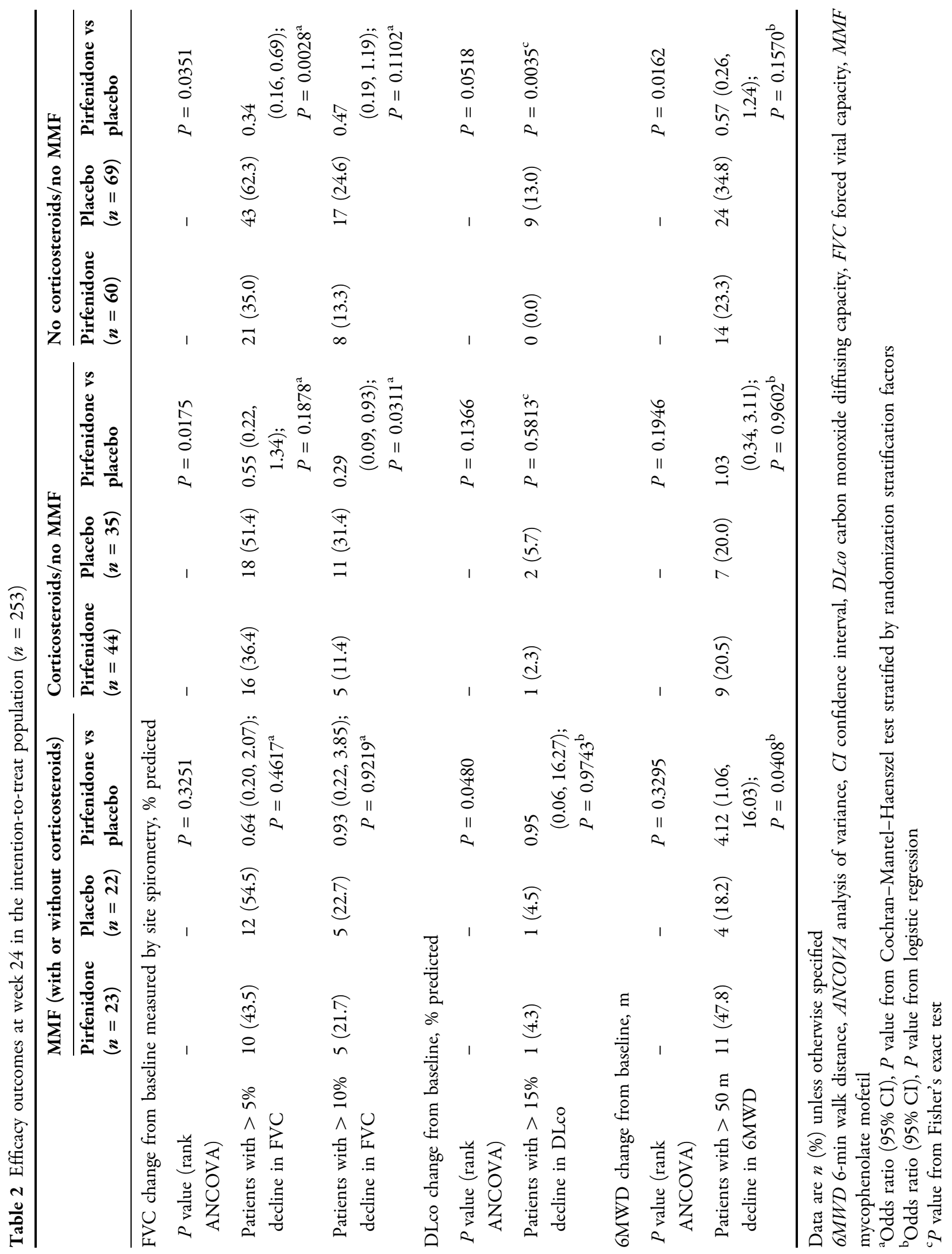




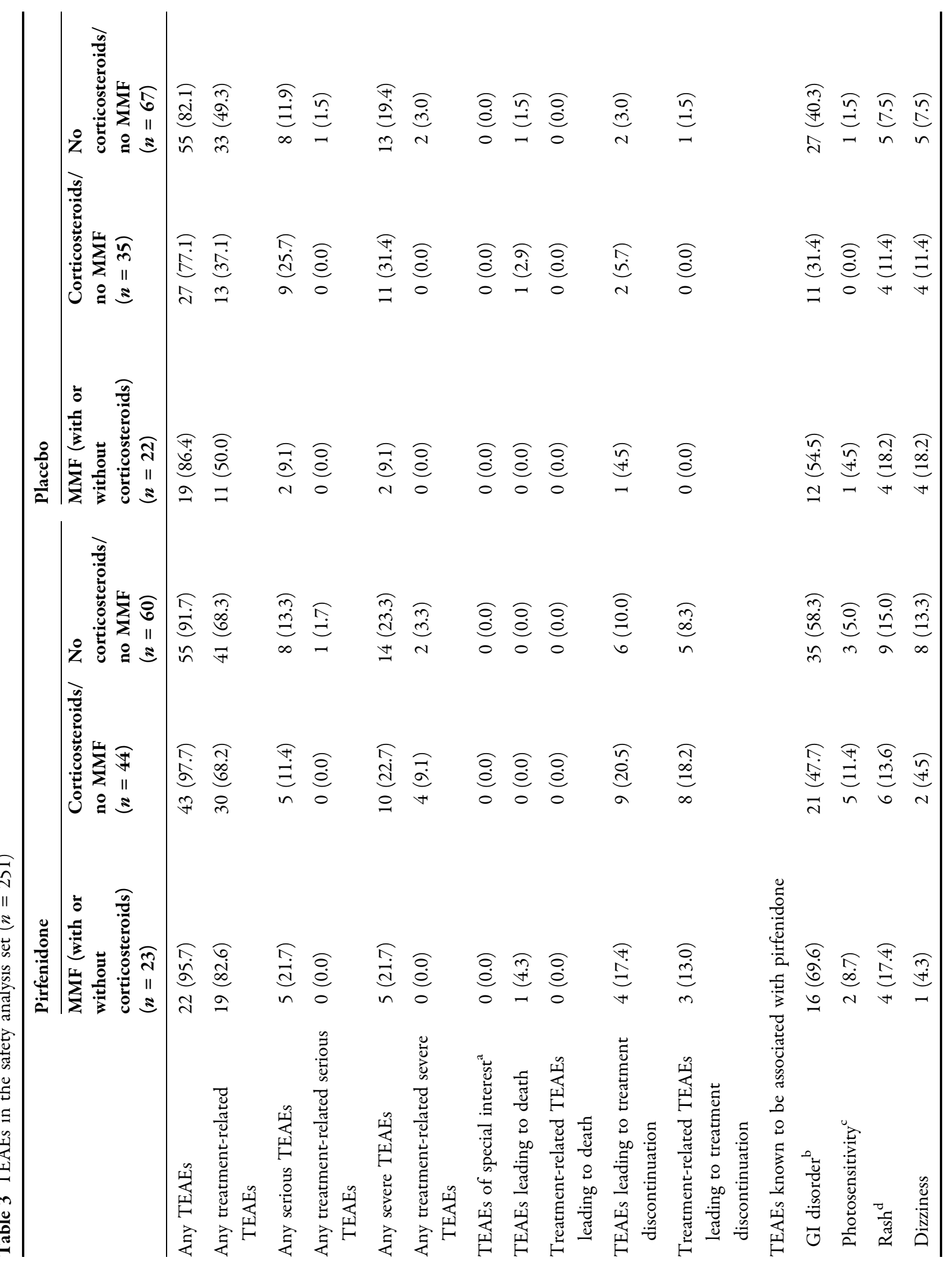




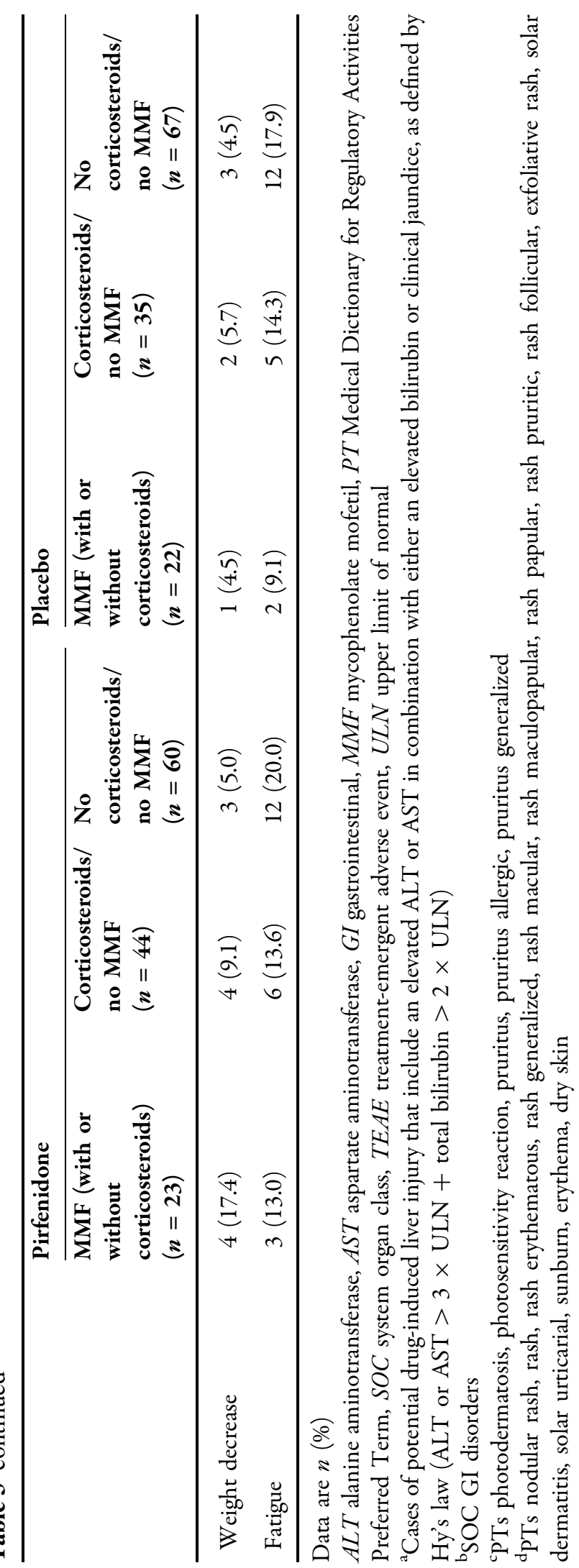


such, more research and accurate disease genotyping and phenotyping are required.

The SENSCIS study of nintedanib in systemic sclerosis-associated (SSc)-ILD suggested that MMF may have an additive effect in reducing FVC decline [11]; however, the present study indicated that pirfenidone may be less effective with concomitant MMF use. This may be because SENSCIS was in a patient population with SSc-ILD, since immunomodulators including MMF have been shown to benefit this population as a result of the autoimmune nature of the disease $[20,21]$. However, uILD populations are more heterogeneous than those with a specific ILD diagnosis, such as SSc-ILD [4], so immunomodulators may not be consistently beneficial in this population. For example, it has been previously suggested that treatment with the immunomodulator cyclophosphamide may be beneficial for patients with IPAF but may be less effective in patients with non-IPAF uILD [8]. Moreover, the PANTHER-IPF trial showed that treatment with immunomodulators (a combination of prednisone, azathioprine, and $\mathrm{N}$-acetylcysteine) was associated with detrimental outcomes including increased rate of death and hospitalization versus placebo in patients with IPF [22]. Considering that some patients with uILD have a similar disease course to IPF again suggests that immunomodulators may not benefit all patients with uILD. It is important to note that a greater proportion of patients $(48.4 \%)$ in SENSCIS were receiving MMF compared with the present study $(17.8 \%)$ and that previous corticosteroid use was not investigated in SENSCIS [11].

Unlike the present study, the INBUILD study reported that the introduction of immunomodulatory therapies, including MMF, during the study did not affect the benefit of nintedanib in patients with progressive fibrosing non-IPF ILD on reducing FVC decline versus placebo, although fewer patients were receiving MMF in INBUILD than in the present study (2.7\% vs $17.8 \%$ [at randomization]) since this was a restricted therapy in INBUILD [12]. Moreover, INBUILD reported that the use of glucocorticoids at baseline (54.4\% of patients) did not affect the benefit of nintedanib on reducing FVC decline versus placebo, although it should be noted that high-dose (greater than $20 \mathrm{mg} /$ day) glucocorticoids were restricted [12]; similarly, the present study showed a beneficial treatment effect for pirfenidone on FVC change in patients not taking MMF regardless of previous corticosteroid use (31.2\% of total patients). Likewise, the RELIEF trial showed that pirfenidone treatment was efficacious in patients with other non-IPF progressive fibrosing ILDs, the majority of whom were receiving immunomodulators at baseline $(73 \%$ and $89 \%$ in the pirfenidone and placebo groups, respectively) [18].

The safety profile of pirfenidone versus placebo was generally similar between all the subgroups. The incidence of treatment-related TEAEs was higher in the MMF subgroup than the no-corticosteroids/no-MMF subgroup in pirfenidone-treated patients; this increase in treatment-related TEAEs appears to be driven by an increase in mild-to-moderate GI disorders in the MMF subgroup. The LOTUSS trial of pirfenidone in SSc-ILD also found that the safety profile of pirfenidone was comparable in the patients who did or did not take concomitant MMF; however, there was a lower proportion of patients reporting severe TEAEs or TEAEs leading to discontinuation in the MMF versus noMMF subgroup [23], which was not the case in the current study.

Limitations of the present analyses include the low patient numbers, meaning that these data should be interpreted with caution and firm conclusions cannot be drawn. In addition, the analysis by corticosteroid use was a post hoc analysis (leading to subgroups of different sizes as a result of corticosteroid use not being a stratification factor, unlike the analysis by MMF use). The analysis by MMF use was restricted to use of MMF treatment at randomization, whereas the analysis by corticosteroid use included all patients who started corticosteroids prior to or at baseline. Therefore, it is unclear whether patients in the MMF or corticosteroid subgroups received these treatments throughout the study. Furthermore, no clinical information relating to the reasons for treating patients with MMF was available, but it is possible that the clinical features of patients who 
received MMF were different to those who did not. Additionally, patients with uILD are a heterogeneous population [3]; some have a predominantly fibrotic phenotype like IPF whereas others have a predominantly inflammatory phenotype (including patients with IPAF) or a combination of both fibrotic and inflammatory phenotypes, which may mean that the effect of pirfenidone treatment varies on a case-by-case basis. It should be noted that the study included patients who did not have a biopsy, which is reflective of clinical reality where many patients will not or cannot have a biopsy. It is therefore possible that some of these patients may have had an underlying pathological pattern of usual interstitial pneumonia, which could have led to a diagnosis of IPF if they had a biopsy. Although, it is important to note that even patients with a low-confidence IPF diagnosis were excluded from the study. However, the exclusion of patients with a low-confidence IPF diagnosis, as well as patients who received corticosteroids after baseline, may have contributed to the small sample size, and the patients who were included in the current analyses may not be reflective of patients with uILD treated during real-world clinical practice. A further limitation of these analyses is that patients were not permitted to receive any other immunomodulators during the trial. Although MMF and corticosteroids are the common agents of choice, other immunomodulators may also be used to treat patients with uILD (e.g., cyclophosphamide); as such, further evaluation including a broader range of immunomodulators is required.

\section{CONCLUSION}

Although limited by design and small sample sizes, this subgroup analysis suggests that pirfenidone may be less effective in patients with UILD receiving MMF at randomization, whereas a beneficial treatment effect for pirfenidone on FVC change was observed in patients not receiving MMF at randomization regardless of previous corticosteroid use. Pirfenidone was well tolerated in patients with UILD regardless of MMF and/or corticosteroid use. Further research is needed to explore these findings in a larger group of patients in a study appropriately designed to determine the effects of concomitant immunomodulators with pirfenidone in patients with progressive fibrosing UILD.

\section{ACKNOWLEDGEMENTS}

We would like to thank the patients, their family members, and participating staff at all of the study centers.

Funding. This study is sponsored by $F$. Hoffmann-La Roche, Ltd., Basel, Switzerland. This trial was initially developed as part of an NIHR-funded Clinician Scientist Fellowship awarded to Toby M. Maher (NIHR reference CS2013-13-017) and subsequently adapted following discussions with F. Hoffmann-La Roche, Ltd. The Advances in Therapy Rapid Service Fees and Open Access Fees are funded by F. Hoffmann-La Roche, Ltd.

Medical Writing, Editorial, and Other Assistance. Medical writing support was provided by Adele Edwards, PhD, of CMC AFFINITY, McCann Health Medical Communications, funded by F. Hoffmann-La Roche, Ltd.

Prior Presentation. Some of the results of the study were presented as a poster at the virtual American Thoracic Society Congress 2020 (August 5-10, 2020).

Authorship. All named authors meet the International Committee of Medical Journal Editors (ICMJE) criteria for authorship of this article, take responsibility for the integrity of the work as a whole, and have given their approval for this final version to be published.

Author Contributions. All authors were involved in the conception and/or design of the work and interpretation of study results, contributed to the brief report from the outset, and read and approved the final draft. Data analysis was performed by FG. All authors vouch for the 
accuracy of the content included in the final brief report.

Disclosures. Michael Kreuter has received fees for speaking and/or organizing education from AstraZeneca, Bayer, Boehringer Ingelheim, Chiesi, ERS, F. Hoffmann-La Roche, Ltd., GlaxoSmithKline, and Novartis; has received fees for consulting from Boehringer Ingelheim, F. Hoffmann-La Roche, Ltd., Galapagos, GlaxoSmithKline, and InterMune; and has received research funding, including institutional funding, from BMBF, Boehringer Ingelheim, DFG, the German Center for Lung Research (DZL), F. Hoffmann-La Roche, Ltd., Hopp Stiftung, InterMune, Lilly, Lungenfibrose E.V., Medac, Olympus, UKGM, and WATL. Toby M. Maher is supported by an NIHR Clinician Scientist Fellowship (NIHR reference CS-201313-017) and a British Lung Foundation Chair in Respiratory Research (C17-3); has received industry-academic research funding from GlaxoSmithKline R\&D and UCB; has served on a clinical trial advisory board for GlaxoSmithKline R\&D; has received stock options from Apellis; and has received consultancy or speaker fees from AstraZeneca, Bayer, Biogen Idec, Boehringer Ingelheim, Cipla, F. Hoffmann-La Roche, Ltd., GlaxoSmithKline R\&D, ProMetic, Samumed, and UCB. Tamera J. Corte has received unrestricted educational grants, travel assistance, and speaker fees from, and has served on advisory boards for, Boehringer Ingelheim; has received unrestricted educational grants and speaker fees and has served on advisory boards for F. Hoffmann-La Roche, Ltd.; has received unrestricted educational grants from Actelion, Bayer, Galapagos, and Sanofi; and has served on advisory boards for AstraZeneca, Bristol Myers Squibb, and Promedior (now a fully owned subsidiary of F. Hoffmann-La Roche, Ltd.). Maria Molina-Molina, or her institution, has received grants from AstraZeneca, Boehringer Ingelheim, BRN, Chiesi, F. Hoffmann-La Roche, Ltd., GlaxoSmithKline, and InterMune. Judit Axmann and Klaus-Uwe Kirchgaessler are employees and shareholders of F. Hoffmann-La Roche, Ltd. Frank Gilberg is an employee of F. Hoffmann-La Roche, Ltd. Vincent Cottin reports personal fees and non- financial support from Actelion, F. HoffmannLa Roche, Ltd., and Promedior (now a fully owned subsidiary of F. Hoffmann-La Roche, Ltd.); grants, personal fees, and non-financial support from Boehringer Ingelheim; personal fees from AstraZeneca, Bayer/MSD, Celgene/ BMS, FibroGen, Galapagos, Galecto, Novartis, PureTech, RedX, Sanofi, and Shionogi, outside the submitted work.

Compliance with Ethics Guidelines. The trial was done in accordance with the ethical principles of the Good Clinical Practice guidelines and the Declaration of Helsinki, and local laws for countries in which the research was done. Informed consent was obtained from each participant by the study investigator before any study-specific screening procedures were done.

Data Availability. Qualified researchers may request access to individual patient-level data through the clinical study data request platform (https://vivli.org). Further details on Roche's criteria for eligible studies are available here (https://vivli.org/members/ourmembers). For further details on Roche's Global Policy on the Sharing of Clinical Information and how to request access to related clinical study documents, see here (https://www.roche.com/ research_and_development/who_we_are_how_ we_work/clinical_trials/our_commitment_to_ data_sharing.htm).

Open Access. This article is licensed under a Creative Commons Attribution-NonCommercial 4.0 International License, which permits any non-commercial use, sharing, adaptation, distribution and reproduction in any medium or format, as long as you give appropriate credit to the original author(s) and the source, provide a link to the Creative Commons licence, and indicate if changes were made. The images or other third party material in this article are included in the article's Creative Commons licence, unless indicated otherwise in a credit line to the material. If material is not included in the article's Creative Commons licence and your intended use is not permitted by statutory regulation or exceeds the permitted use, you 
will need to obtain permission directly from the copyright holder. To view a copy of this licence, visit http://creativecommons.org/licenses/by$\mathrm{nc} / 4.0 /$.

\section{REFERENCES}

1. Ryerson CJ, Collard HR. Update on the diagnosis and classification of ILD. Curr Opin Pulm Med. 2013;19:453-9.

2. Cottin V, Hirani NA, Hotchkin DL, et al. Presentation, diagnosis and clinical course of the spectrum of progressive-fibrosing interstitial lung diseases. Eur Respir Rev. 2018;27:180076.

3. Guler SA, Ellison K, Algamdi M, Collard HR, Ryerson CJ. Heterogeneity in unclassifiable interstitial lung disease. A systematic review and meta-analysis. Ann Am Thorac Soc. 2018;15:854-63.

4. Skolnik K, Ryerson CJ. Unclassifiable interstitial lung disease: a review. Respirology. 2016;21:51-6.

5. Fischer A, Antoniou KM, Brown KK, et al. An official European Respiratory Society/American Thoracic Society research statement: interstitial pneumonia with autoimmune features. Eur Respir J. 2015;46: 976-87.

6. Chartrand S, Swigris JJ, Stanchev L, Lee JS, Brown KK, Fischer A. Clinical features and natural history of interstitial pneumonia with autoimmune features: a single center experience. Respir Med. 2016;119:150-4.

7. McCoy SS, Mukadam Z, Meyer KC, et al. Mycophenolate therapy in interstitial pneumonia with autoimmune features: a cohort study. Ther Clin Risk Manag. 2018;14:2171-81.

8. Wiertz IA, van Moorsel CHM, Vorselaars ADM, Quanjel MJR, Grutters JC. Cyclophosphamide in steroid refractory unclassifiable idiopathic interstitial pneumonia and interstitial pneumonia with autoimmune features (IPAF). Eur Respir J. 2018;51: 1702519 .

9. Sasano H, Hagiwara E, Kitamura $\mathrm{H}$, et al. Long-term clinical course of anti-glycyl tRNA synthetase (antiEJ) antibody-related interstitial lung disease pathologically proven by surgical lung biopsy. BMC Pulm Med. 2016;16:168.

10. Leung SC, Churg AM, Leipsic JA, Levy RD, Wilcox PG, Ryerson CJ. Unclassifiable interstitial lung disease: an unresolved diagnostic dilemma. Respirol Case Rep. 2015;3:85-8.
11. Distler O, Highland KB, Gahlemann M, et al. Nintedanib for systemic sclerosis-associated interstitial lung disease. N Engl J Med. 2019;380:2518-28.

12. Cottin V, Richeldi L, Rosas I, et al. Nintedanib and immunomodulatory therapies in progressive fibrosing interstitial lung diseases. Respir Res. 2021;22:84.

13. Wijsenbeek M, Kreuter M, Olson A, et al. Progressive fibrosing interstitial lung diseases: current practice in diagnosis and management. Curr Med Res Opin. 2019;35:2015-24.

14. Flaherty KR, Wells AU, Cottin V, et al. Nintedanib in progressive fibrosing interstitial lung diseases. N Engl J Med. 2019;381:1718-27.

15. Maher TM, Corte TJ, Fischer A, et al. Pirfenidone in patients with unclassifiable progressive fibrosing interstitial lung disease: a double-blind, randomised, placebo-controlled, phase 2 trial. Lancet Respir Med. 2020;8:147-57.

16. Richeldi L, du Bois RM, Raghu G, et al. Efficacy and safety of nintedanib in idiopathic pulmonary fibrosis. N Engl J Med. 2014;370:2071-82.

17. King TE Jr, Bradford WZ, Castro-Bernardini S, et al. A phase 3 trial of pirfenidone in patients with idiopathic pulmonary fibrosis. $\mathrm{N}$ Engl J Med. 2014;370:2083-92.

18. Behr J, Prasse A, Kreuter $M$, et al. Pirfenidone in patients with progressive fibrotic interstitial lung diseases other than idiopathic pulmonary fibrosis (RELIEF): a double-blind, randomised, placebocontrolled, phase $2 \mathrm{~b}$ trial. Lancet Respir Med. 2021;9:476-86.

19. Maher TM, Corte TJ, Fischer A, et al. Pirfenidone in patients with unclassifiable progressive fibrosing interstitial lung disease: design of a double-blind, randomised, placebo-controlled phase II trial. BMJ Open Respir Res. 2018;5:e000289.

20. Tashkin DP, Elashoff R, Clements PJ, et al. Cyclophosphamide versus placebo in scleroderma lung disease. N Engl J Med. 2006;354:2655-66.

21. Tashkin DP, Roth MD, Clements PJ, et al. Mycophenolate mofetil versus oral cyclophosphamide in scleroderma-related interstitial lung disease (SLS II): a randomised controlled, doubleblind, parallel group trial. Lancet Respir Med. 2016;4:708-19.

22. Raghu G, Anstrom KJ, King TE Jr, Lasky JA, Martinez FJ. Prednisone, azathioprine, and N-acetylcysteine for pulmonary fibrosis. N Engl J Med. 2012;366: 1968-77. 
23. Khanna D, Albera C, Fischer A, et al. An open-label, phase II study of the safety and tolerability of pirfenidone in patients with scleroderma-associated interstitial lung disease: the LOTUSS trial. J Rheumatol. 2016;43:1672-9. 